\title{
Periapical Inflammation Affecting Coronally- inoculated Dog Teeth with Root Fillings Augmented by White MTA Orifice Plugs
}

\author{
Terence Mah, DDS, MS, Bettina Basrani, DDS, PhD, João M. Santos, DDS, MS, \\ Elizeu A. Pascon, DDS, MSD, PhD, Leo Tjäderhane, DDS, PhD, Ghassan Yared, DDS, MSc, \\ Herenia P. Lawrence, DDS, PhD, and Shimon Friedman, DMD
}

\begin{abstract}
Placement of orifice plugs has been suggested to augment the seal of conventional root canal fillings. This study assessed in vivo the efficacy of white mineral trioxide aggregate (MTA) plugs in preventing periapical inflammation subsequent to coronal inoculation of root-filled teeth. The tworooted mandibular premolars of six beagle dogs were conventionally prepared and filled with guttapercha and sealer. A white MTA orifice plug was placed into one canal in each tooth. Pulp chambers were inoculated with plaque except for 12 teeth (negative control), and restored. Radiographs were taken at regular intervals. At $\mathbf{1 0}$ months, dogs were killed and jaw blocks processed for histology. None of the roots revealed radiographic or histologic evidence of severe inflammation. Mild inflammation was observed in $17 \%$ and $39 \%$ of the roots with and without an orifice plug, respectively (McNemar, $p>0.05$ ). Without development of severe inflammation, the seal augmentation efficacy of MTA orifice plugs could not be determined.
\end{abstract}

Infection of the root canal system by oral microorganisms results in the development of apical periodontitis (1); therefore, the objective of apical periodontitis therapy is to exclude microorganisms. Routinely, the root canal system is disinfected to eliminate existing microorganisms. The canals are then filled and the tooth restored to prevent ingress of microorganisms subsequent to treatment. Despite these elaborate procedures, microorganisms can still infect the filled root canal system $(2,3)$. They can penetrate via the coronal access cavity (4), lateral canals, or the dentinal tubules (5), and propagate to establish an infective process resulting in posttreatment apical periodontitis $(6,7)$. Because the currently used root filling materials do not effectively resist microbial ingress (7), each time the canals of root-filled teeth become exposed to the oral environment there is a risk of developing infection and consequent posttreatment apical periodontitis (3). This risk may even be greater when the root filling is partially removed for post placement and the seal is thus further compromised (8). Augmentation of the root canal seal is, therefore, a desirable clinical goal because it may improve the outcome of root canal treatment (4).

The root canal seal may be augmented with a different material than the root filling. One suggestion has been to seal the pulp chamber floor with a dentin-bonding restorative material, such as glass-ionomer cement (4) or composite resin (9). However, such a procedure is impractical in canals designated for post placement, or when extension of the core into the canal orifices is required to improve retention. Alternatively, it has been suggested that the root canal seal can be augmented by means of an orifice plug placed over the root-filling material (10). The properties of the plug material should include a bacteria-tight seal, compatibility with root filling and restorative materials, easy placement, and retreatability. In their clinical study, Sjögren et al. (11) discussed using zinc-oxide eugenol (ZOE) as a plug, without referring to its efficacy. However, when tested as a pulpal floor seal in vitro, ZOE has shown significant leakage (9). Recently, mineral trioxide aggregate (MTA) (ProRoot MTA, Dentsply, Tulsa, OK) has been introduced as a sealing compound for endodontic applications. Because MTA has demonstrated excellent sealing ability against microorganisms, both in vitro and in vivo, it has been suggested for use as a root canal orifice plug in conjunction with the conventional root filling (10).

One concern regarding the use of MTA as an orifice plug has been the dark gray color of the material that may compromise the esthetic appearance of the treated teeth. This concern resulted in the recent introduction of white MTA, intended to eliminate discoloration of teeth and surrounding tissues. The purpose of this study was to assess in vivo the efficacy of white MTA, placed as an orifice plug to augment conventional root fillings, in preventing periapical inflammation subsequent to coronal inoculation with oral microorganisms. It was hypothesized that the incidence of inflammation affecting the roots with MTA-augmented fillings would be at least 30\% less than that for roots with just the conventional fillings.

\section{MATERIALS AND METHODS}

The study methodology and sample size estimation were based on the model established by Friedman et al. $(6,7)$. With an 
TABLE 1. Periapical inflammation around filled roots of mandibular premolars of dogs 10 months after inoculation of the pulp chamber (where applied)

\begin{tabular}{|c|c|c|c|c|c|}
\hline \multirow[t]{2}{*}{ Group } & \multirow[t]{2}{*}{ Inoculation } & \multirow[t]{2}{*}{ No. of Roots } & \multirow[t]{2}{*}{ Treatment } & \multicolumn{2}{|c|}{$\begin{array}{c}\text { Periapical } \\
\text { Inflammation } \\
\text { (Roots) }\end{array}$} \\
\hline & & & & None & Mild \\
\hline \multicolumn{6}{|c|}{ Experimental } \\
\hline 1 & Yes & $24(23)^{\star}$ & root filling + MTA plug & 19 & 4 \\
\hline 2 & Yes & $24(23)^{\star}$ & root filling alone & 14 & 9 \\
\hline
\end{tabular}

* Number in parentheses represents samples available for analysis after exclusion of exposed roots and those damaged in histology.

expected differential outcome of $30 \%$, sample size was set at 24 roots per group, for statistical power of $80 \%$ and a $5 \%$ significance level. Accordingly, six, male, 3-year-old, beagle dogs were included. University of Toronto Animal Care Committee approved the study protocol, and the handling and maintenance of the dogs strictly conformed to the guidelines of the Canadian Council on Animal Care. For all clinical procedures, the dogs were anesthetized with $25 \mathrm{mg} / \mathrm{kg}$ of thiopental, followed by $\mathrm{O}_{2}$ and $2 \%$ isoflurane inhalation.

In all dogs, root canal treatment was performed in the six two-rooted mandibular premolars under strict aseptic conditions. Before treatment, the teeth were radiographed, pumiced, isolated with rubber dam, and thoroughly wiped with $10 \%$ povidone-iodine solution. The central cusp of each tooth was slightly reduced and a mesiodistal access cavity prepared with a sterile bur at highspeed, under sterile saline irrigation. The root canals were cleaned and shaped with ProFile rotary files (Dentsply-Tulsa Dental) to at least size 40 at the working length. Each canal was irrigated intermittently with $10 \mathrm{ml}$ of $2.5 \% \mathrm{NaOCl}$ and finally rinsed with 3 $\mathrm{ml}$ of $17 \%$ EDTA, followed by $3 \mathrm{ml}$ of sterile saline. The canals were then blotted dry with sterile paper points and filled with Roth's 801 root canal cement (Roth International Ltd., Chicago, IL) and laterally condensed gutta-percha. The coronal excess of the root filling was removed with a heat carrier (Touch n' Heat, Analytic Technology, Orange, CA) and the gutta-percha at the orifice compacted with a plugger. Two of three treated premolars on one side of each dog were randomly assigned, by the roll of a dice, to the experimental groups. These groups were paired within each treated tooth as follows (Table 1): in one root, the coronal 2 $\mathrm{mm}$ of the root filling was removed and replaced with a 2-mm white MTA plug (group 1); in the second root, the root filling was left intact (group 2). A sterile sponge pellet moistened with sterile water (required to facilitate the setting of MTA) was placed over each of the canal orifices and the access cavities sealed with a compomer restorative material (Dyract, Dentsply, York, PA). The remaining premolar on the same side served as the negative control, with its canals receiving similar treatment as in the experimental groups. The premolars on the contralateral side were similarly assigned; however, the grouping of the canals in each tooth was reversed mesiodistally to obtain equal distribution of mesial and distal roots in either group. Total randomization was precluded by the requirement to pair both treatments in each tooth.

After 1 week, the access cavities in the teeth assigned to the experimental groups were reaccessed and the sponge pellets removed. The pulp chambers were inoculated with isologous plaque scaled from the dog's teeth. All teeth were then resealed with a sterile sponge pellet and Dyract. The pulp chambers of the teeth in the negative control group were not reaccessed and remained undisturbed.

To facilitate accurate radiographic monitoring of the teeth, impressions were taken of the dog's lower jaw and stents fabricated. All teeth were radiographed 5 days after inoculation, and then at 3, 6, and 10 months. Additional radiographs were taken at 7, 8, and 9 months, of two different dogs each time, and observed for emergence of apical periodontitis. Radiographs were exposed and processed in a standard manner.

To prevent the dogs from biting on hard objects, the housing cage was lined with Plexiglas sheets. Soft toys were provided for play. During the observation period, dogs were monitored daily for signs of distress, as manifested by not eating, not sleeping, pawing the mouth area, or depressed behavior. The teeth were examined each month to verify the integrity of the restorations.

The experiment was terminated 10 months after inoculation. The dogs were anesthetized with thiopental and killed by bilateral head perfusion with $10 \%$ phosphate-buffered formalin. Before removal of blocks for histology, four teeth assigned to the experimental groups in different dogs were randomly chosen for culturing of the sponge pellet in the pulp chamber. The tooth was isolated, pumiced, and the area wiped with $10 \%$ povidone-iodine solution. The occlusal filling was reduced with a sterile bur to a thin layer, the tooth surface wiped again with povidone iodine, and the remaining Dyract removed with a sterile spoon excavator. The sponge pellet was retrieved with sterile forceps and immediately placed in a vial containing $1 \mathrm{ml}$ of reduced transport fluid. The vials were promptly processed for aerobic and anaerobic cultures.

The dog mandibles were separated, block dissected from first premolar to first molar, post-fixed in phosphate-buffered formalin, and decalcified in 5\% formic acid. The crowns of the teeth were sectioned off above the cervical level. Each block was divided between the roots of the third premolar. The resulting smaller blocks were trimmed, including horizontal reduction to a level immediately apical to the MTA plugs, embedded in paraffin, and serially sectioned $(6-\mu \mathrm{m}$ thick) along the sagittal plane. Three of every 12 sections were mounted on glass slides and stained, two with hematoxylin and eosin and one with Brown and Brenn. The periradicular tissues were examined histologically under a light microscope.

Periradicular inflammation was rated in accordance with previous studies $(6,7)$ as follows: (1) none: normal appearance of the root surface and surrounding tissues; (2) mild: localized inflammatory cell infiltrate without bone and root resorption; and (3) severe: diffuse infiltrate associated with bone or root resorption. McNemar paired analysis was used to compare the incidence of inflammation in the two experimental groups and the two negative 
TABLE 2. McNemar test for paired proportions in teeth inoculated with oral plaque (group 1 versus group 2)

\begin{tabular}{|c|c|c|c|c|}
\hline & & \multicolumn{2}{|c|}{ Root filling alone (group 2) } & \\
\hline & & No inflammation & Mild inflammation & \\
\hline \multirow[t]{4}{*}{$\begin{array}{l}\text { Root filling + MTA } \\
\text { plug (group 1) }\end{array}$} & No inflammation & $\begin{array}{l}13 \\
57 \%\end{array}$ & $\begin{array}{c}6 \\
26 \%\end{array}$ & $\begin{array}{c}\text { no. of roots } \\
\% \text { of total }\end{array}$ \\
\hline & Mild inflammation & 1 & 3 & no. of roots \\
\hline & & $4 \%$ & $13 \%$ & $\%$ of total \\
\hline & Total & \multicolumn{2}{|c|}{$\begin{array}{c}23 \\
100 \%\end{array}$} & \\
\hline
\end{tabular}

McNemar Chi-square test $=2.29 ; \mathrm{df}=1 ; \mathrm{p}=0.125$

Odds ratio $=6$.

controls. Chi-square test was used to compare each experimental group with its corresponding control. Significance was established at the $5 \%$ level.

\section{RESULTS}

All dogs tolerated the operative procedures well, and none demonstrated signs of distress throughout the observation period. Clinically, there was no evidence of swelling or a sinus tract associated with any of the treated teeth. Despite a rigorous attempt to prevent excessive chewing and trauma to the treated teeth by controlling the dogs' environment, 10 of the original 72 fillings were lost between the sixth and the tenth months. None of the affected teeth could be restored again. The one contaminated tooth from the negative control (filling lost at 6 months) was excluded from the analysis, whereas contamination in the nine experimentally treated teeth was considered to have no impact, because it equally affected both groups, and the exposed teeth were intentionally inoculated with oral microorganisms (7).

One block from dog 4 containing three roots (one tooth from the negative control and a root from group 2) was inadvertently damaged during histologic processing. These roots and the paired root from group 1 also were excluded from the analysis. Thus, the number of pairs of roots available for analysis of the experimental and negative control groups was 23 and 10, respectively.

Radiographic observations at all time intervals revealed intact periodontal ligament spaces without noticeable changes in the apical bone pattern. The histological outcomes in all groups are summarized in Table 1. There was no severe inflammation about any of the treated roots. Mild inflammation was observed in $17 \%$ of the roots in group 1 and in $39 \%$ of the roots in group 2. This difference between the two experimental groups was not statistically significant (Table 2). In the negative control, mild inflammation was observed in $30 \%$ of the roots, with equal distribution among the roots with and without an orifice plug $(p=1.00)$. The difference between each experimental group and its corresponding negative control was not significant. Aerobic and anaerobic cultures of the recovered sponge pellets from four experimental teeth were all positive for microbial growth. Brown and Brenn staining was negative for the presence of microbial colonies in all of the roots.

\section{DISCUSSION}

The experimental model used in this study was originally designed to assess the efficacy of root filling materials with regard to their long-term function: the prevention of coronal microbial ingress and consequent development of apical periodontitis (6). It simulates coronal leakage of microorganisms, which is a clinical condition that can lead to posttreatment apical periodontitis (4), while controlling other variables that might influence the outcome of treatment. Using this clinical simulation, the present study compared the efficacy of a conventional root filling (gutta-percha and sealer) either alone or when augmented with a white MTA orifice plug. Three modifications to the original design were introduced: first, rotary files were primarily used instead of hand files $(6,7)$ to facilitate the cleaning and shaping procedure. Second, apical enlargement was more extensive. Originally, all canals were arbitrarily enlarged to size 40 (7); however, we frequently noted in the third and fourth premolars that after enlargement to size 40 files were still not binding and bleeding persisted. The bleeding canals were further enlarged, occasionally to size 80 , before files were binding and the bleeding stopped. Third, compomer was used to seal the access. Originally glass-ionomer cement was used (6); however, to avoid concerns regarding its antimicrobial activity, it was later substituted for amalgam (7). The amalgam fillings did not last the 6 months of the study (7); therefore, a compomer was selected that would adhere to the tooth structure and thus reduce the risk of filling loss. Loss of fillings was considerably less frequent in this study than in the previous one (7), although not totally avoidable. Fourth, a positive control was not included. Because the positive controls in the previous studies $(6,7)$ inoculated teeth with instrumented but unfilled canals- had invariably developed extensive, severe periapical inflammation, and similar results have been reported by others (12), it was deemed unnecessary to expose the dogs to undue discomfort and infection by repeating the positive control.

We hypothesized that at least $20 \%$ of the roots filled conventionally without MTA plugs (group 2) would develop severe inflammation, in accordance with the $21 \%$ incidence found for similarly filled roots in the previous 6-month study (7). Therefore, the absence of radiographic signs of apical periodontitis and severe inflammation in the present 10 -month study was unexpected. However, in our preliminary 14-week study (6), none of the nine similarly filled roots were affected by severe inflammation either (Table 3). When considered cumulatively, the findings in the three animal studies sharing the same methodology seem to corroborate the clinical report by Ricucci et al. (13). They observed root-filled teeth that had been exposed to the oral environment for up to 4 years without periapical deterioration and suggested that welltreated teeth could resist coronal leakage and subsequent apical periodontitis. Nevertheless, absence of radiographic signs of apical periodontitis does not rule out the presence of inflammation $(6,7)$.

Indeed, 19 roots in the present study were associated with mild inflammation. Interpretation of mild inflammation remains speculative, as in the previous studies $(6,7)$, with three possible explanations: (a) persistent tissue response to cleaning and shaping; (b) 
TABLE 3. Periapical inflammation around roots filled with Roth's 801 sealer and gutta-percha in previous studies, at 14 weeks (6) and 6 months (7)

\begin{tabular}{|c|c|c|c|c|c|}
\hline \multirow{2}{*}{$\begin{array}{l}\text { Observation } \\
\text { Period }\end{array}$} & \multirow{2}{*}{ Inoculation } & \multirow{2}{*}{ No. of Roots } & \multicolumn{3}{|c|}{ Periapical Inflammation } \\
\hline & & & None & Mild & Severe \\
\hline 14 weeks & Yes & 9 & 3 & 6 & 0 \\
\hline \multirow[t]{2}{*}{6 months } & Yes & 24 & 13 & 6 & 5 \\
\hline & $\mathrm{No}^{*}$ & 11 & 7 & 3 & 1 \\
\hline
\end{tabular}

* Invalidated as a negative control because of lost fillings and subsequent contamination with oral fluids.

persistent tissue response to the root-filling materials; and (c) early stage of emerging apical periodontitis. Friedman et al. (6) suggested that the mild inflammation observed in their short-term study of 14 weeks could represent tissue reaction to the treatment procedure. This suggestion was supported by the fact that mild inflammation also affected $33 \%$ of the roots in the negative control. In the 14-week period inflammation associated with severing the pulp would have subsided (14), and so would the initially acute inflammatory reaction caused by the ZOE-based Roth's 801 sealer (15); however, because ZOE may disintegrate into tissue-irritating components (16), the sealer could have sustained a low-grade chronic inflammation.

In the longer study of 6 months, Friedman et al. (7) suggested that for the major part, the mild inflammation represented an early stage of apical periodontitis that put it in the same adverse category as severe inflammation. This suggestion seemed to be supported by the considerable reduction in the incidence of mild inflammation, from $67 \%$ at 14 weeks (6) to $25 \%$ at 6 months (for roots filled with Roth's 801 sealer and condensed gutta-percha) (7). Further support was derived from the fact that the incidence of mild inflammation in the two experimental groups ( $25 \%$ for canals filled using Roth's 801 sealer and $8 \%$ for canals sealed with an experimental sealer) was almost identical to the incidence of severe inflammation in the same groups $(21 \%$ and $8 \%$, respectively), suggesting that they represented different stages of the same entity. However, it should be noted that the negative control in the 6-month study (7) was incapacitated by the loss of fillings. Without the ability to compare the experimental and negative control groups, the suggestion that the mild inflammation represented emerging apical periodontitis could not be validated.

In the present study (Roth's 801 sealer used in all canals) mild inflammation affected $28 \%$ of the roots, much less than in the 14-week study (6) and comparable to the similarly filled roots in the 6-month study (7). Most importantly, in the present study also $30 \%$ of roots in the negative control showed mild inflammation although they were not inoculated. Because it is unlikely that all 10 teeth in the negative control had become contaminated, the mild inflammation in some or all of the affected roots must have represented tissue reactions to the treatment procedure and not the inoculation, contrary to our previous suggestion (7). Indeed, Brown and Brenn staining did not demonstrate bacterial colonies in any of the roots affected by the mild inflammation. This finding corroborated the preliminary study (6), where bacterial colonization was demonstrated in $92 \%$ of the roots affected by severe inflammation, but in none of the roots with mild inflammation.

This does not rule out the possibility that in some roots, microbial ingress had occurred. However, even in the presence of root canal microorganisms, apical periodontitis may not develop if the root-canal environment does not support growth of specific virulent strains $(12,17)$. Indeed, in a clinical study of root-filled teeth, microorganisms have been found in $45 \%$ of roots that had no signs of apical periodontitis (2). In the end point of the present study, the inoculum was confirmed to be still viable. Nevertheless, viability is not an indication of the inoculating microorganisms' virulence and ability to penetrate the filled canals and establish an infective process.

The absence of severe inflammation calls for consideration of the methodological modifications made in this study. Regarding the cleaning and shaping procedure, the use of rotary files should not influence the results, because in relatively straight canals, such as are present in dogs' premolars, there is no significant difference in the debridement achieved with rotary and hand files (18). The extensive enlargement could have rendered the canals cleaner, with better root-filling adaptation as a result and, potentially, an improved seal. However, tissue debris were still histologically evident in the apical canal portion of several roots (7). Also the use of Dyract for sealing the access cavities should not influence the results, because it does not possess antimicrobial properties (19), as also confirmed by the viability of the inoculum at the end point of the study.

Placing a root canal orifice plug $(10,11)$ offers an advantage over sealing the pulp chamber floor (4), because it can also be placed in canals prepared for post space. If retreatment becomes indicated, the 2- to 4-mm plug can be easily removed. Torabinejad and Chivian (10) suggested the use of MTA for this purpose, based on the material's proven sealing ability against microbial penetration. The newest, white formulation of MTA was found to be suitable for use as an orifice plug. It was easily manipulated and compacted into the canal orifices. No discoloration occurred in any of the teeth, confirming the improved esthetics that can be achieved with the use of this modified material. The shade of the material still offers enough contrast with dentin, so that it can be easily identified if retreatment becomes necessary. One disadvantage of MTA, both the gray and white formulations, is the lengthy setting time and the moisture required to enhance setting. This suggests that restoration of the tooth cannot be performed immediately after the placement of the MTA plug.

This study did not demonstrate significantly different outcomes for the conventional root fillings and those augmented with white MTA, even if the mild inflammation was considered an early stage of apical periodontitis. Thus, a benefit of placing white MTA plugs was not demonstrated. This finding, however, cannot be projected to suggest the long-term effectiveness of this procedure in preventing apical periodontitis subsequent to coronal contamination of root-filled teeth. Longer animal studies are needed to provide insight into the long-term benefits of white MTA orifice plugs. The length of time required for apical periodontitis to emerge subsequent to exposure of the root canal filling to the oral environment remains elusive. Our previous two studies seemed to suggest a time of 3 (6) to 6 months (7). The present study, however, suggested that 
experimentally, canals filled with gutta-percha and Roth's 801 sealer might resist the development of severe periapical inflammation for up to 10 months after they become exposed to oral microorganisms.

This study was supported by a research grant from Dentsply-Tulsa Dental.

The authors thank Dr. Calvin D. Torneck for his help in the development of this study and Ms. Nancy Valliquette for her assistance in the processing of the histology.

Drs. Mah, Pascon, Tjäderhane, Yared, and Friedman are affiliated with the Discipline of Endodontics, and Dr. Lawrence is affiliated with the Discipline of Community Dentistry, Faculty of Dentistry, University of Toronto, Ontario, Canada. Dr. Basrani is affiliated with the Department of Dental Clinical Sciences, Faculty of Dentistry, Dalhousie University, Halifax, Nova Scotia, Canada. Dr. Santos is affiliated with the Department of Dentistry, Faculty of Medicine, University of Coimbra, Portugal. Address request for reprints to Dr. Shimon Friedman, Discipline of Endodontics, Faculty of Dentistry, University of Toronto, 124 Edward Street, Toronto, Ontario M5G 1G6, Canada.

\section{References}

1. Möller AJ, Fabricius L, Dahlen G, Ohman AE, Heyden G. Influence on periapical tissues of indigenous oral bacteria and necrotic pulp tissue in monkeys. Scand J Dent Res 1981;89:475-84.

2. Molander A, Reit C, Dahlen G, Kvist T. Microbiological status of rootfilled teeth with apical periodontitis. Int Endod J 1998;31:1-7.

3. Friedman S. Considerations and concepts of case selection in the management of post-treatment endodontic disease (treatment failure). Endod Topics 2002;1:54-78.

4. Saunders WP, Saunders EM. The root filling and restoration continuumprevention of long-term endodontic failures. Alpha Omegan 1997;90:40-6.

5. Adriaens PA, De Boever JA, Loesche WJ. Bacterial invasion in root cementum and radicular dentin of periodontally diseased teeth in humans. A reservoir of periodontopathic bacteria. J Periodontol 1988;59:222-30.

6. Friedman S, Torneck CD, Komorowski R, Ouzounian Z, Syrtash P, Kaufman A. In vivo model for assessing the functional efficacy of endodontic filling materials and techniques. J Endodon 1997;23:557-61.

7. Friedman S, Komorowski R, Maillet W, Klimaite R, Nguyen HQ, Torneck CD. In vivo resistance of coronally induced bacterial ingress by an experimental glass ionomer cement root canal sealer. J Endodon 2000;26:1-5.

8. Yared GM, Dagher FB, Machtou P. Influence of the removal of coronal gutta-percha on the seal of root canal obturations. J Endodon 1997;23: 146-8.

9. Belli S, Zhang Y, Pereira PN, Pashley DH. Adhesive sealing of the pulp chamber. J Endodon 2001;27:521-6.

10. Torabinejad M, Chivian N. Clinical applications of mineral trioxide aggregate. J Endodon 1999;25:197-205.

11. Sjögren U, Figdor D, Persson S, Sundqvist G. Influence of infection at the time of root filling on the outcome of endodontic treatment of teeth with apical periodontitis. Int Endod J 1997;30:297-306.

12. Pascon EA, Introcaso JH, Langeland K. Development of predictable periapical lesion monitored by subtraction radiography. Endod Dent Traumatol 1987;3:192-208.

13. Ricucci D, Grondahl K, Bergenholtz G. Periapical status of root-filled teeth exposed to the oral environment by loss of restoration or caries. Oral Surg Oral Med Oral Pathol Oral Radiol Endod 2000;90:354-9.

14. Seltzer S, Soltanoff W, Sinai I, Smith J. Biologic aspects of endodontics. IV. Periapical tissue reactions to root-filled teeth whose canals had been instrumented short of their apices. Oral Surg Oral Med Oral Pathol 1969;28: 724-38.

15. Mittal M, Chandra S, Chandra S. Comparative tissue toxicity evaluation of four endodontic sealers. J Endodon 1995;21:622-4.

16. Pascon EA, Leonardo MR, Safavi K, Langeland K. Tissue reaction to endodontic materials: methods, criteria, assessment, and observations. Oral Surg Oral Med Oral Pathol 1991;72:222-37.

17. Sundqvist G. Taxonomy, ecology, and pathogenicity of the root canal flora. Oral Surg Oral Med Oral Pathol 1994;7:522-30.

18. Ahlquist $\mathrm{M}$, Henningsson $\mathrm{O}$, Hultenby $\mathrm{K}$, Ohlin J. The effectiveness of manual and rotary techniques in the cleaning of root canals: a scanning electron microscopy study. Int Endod J 2001;34:533-7.

19. Herrera M, Carrion P, Bravo M, Castillo A. Antibacterial activity of four dentin bonding systems. Int J Antimicrob Agents 2000;15:305-9. 